\title{
Application of various surgical techniques in liver transplantation: a retrospective study
}

\author{
Zhitao Chen ${ }^{1,2,3 \#}$, Weiqiang Ju ${ }^{1,2,3 \#}$, Chuanbao Chen ${ }^{1,2,3}$, Tielong Wang ${ }^{1,2,3}$, Jia Yu ${ }^{1,2,3}$, Xitao Hong ${ }^{1,2,3}$, \\ Yuqi Dong ${ }^{1,2,3}$, Maogen Chen ${ }^{1,2,3}$, Xiaoshun $\mathrm{He}^{1,2,3}$
}

${ }^{1}$ Organ Transplant Center, First Affiliated Hospital of Sun Yat-sen University, Guangzhou, China; ${ }^{2}$ Guangdong Provincial Key Laboratory of Organ Donation and Transplant Immunology, Guangzhou, China; ${ }^{3}$ Guangdong Provincial International Cooperation Base of Science and Technology (Organ Transplantation), Guangzhou, China Contributions: (I) Conception and design: M Chen; (II) Administrative support: M Chen, X He; (III) Provision of study materials or patients: Z Chen, W Ju; (IV) Collection and assembly of data: Z Chen, W Ju, T Wang; (V) Data analysis and interpretation: Z Chen, W Ju, T Wang, J Yu, C Chen, X He, Y Dong; (VI) Manuscript writing: All authors; (VII) Final approval of manuscript: All authors.

\#These authors contributed equally to this work.

Correspondence to: Xiaoshun He; Maogen Chen. Division of Organ Transplant Center, First Affiliated Hospital of Sun Yat-sen University, Guangzhou 510080, China. Email: gdtrc@163.com; chenmg3@mail.sysu.edu.cn.

\begin{abstract}
Background: Surgical techniques of liver transplantation have continually evolved and have been modified. We retrospectively analyzed a single-center case series and compared the advantages and disadvantages of each method.

Methods: Six-hundred and seventy-four recipients' perioperative data were assessed and analyzed stratified by different surgical technics [modified classic (MC), modified piggyback (MPB) and classic piggyback (CPB)]. Results: MELD score and Child-Pugh scores was significantly higher in CPB groups ( $\mathrm{P}=0.008$ and 0.003 , respectively). Anhepatic time in MPB group was longer than those in $\mathrm{CPB}$ group $(\mathrm{P}<0.05)$. The operation duration in MPB group was significantly longer than those in $\mathrm{MC}$ group and $\mathrm{CPB}$ group $(\mathrm{P}=0.003)$. Three patients had outflow obstruction $(\mathrm{P}=0.035)$. The overall survival in MPB group were better than those in MC group and $\mathrm{CPB}$ group in general comparison $(\mathrm{P}<0.001)$. In patients with preoperative creatine $>120 \mu \mathrm{mol} / \mathrm{L}$, the overall survival in $\mathrm{MC}$ group was worst $(\mathrm{P}<0.001)$. In patients with a high MELD score $(>24)$, the overall survival in MPB group tended to be the best $(\mathrm{P}<0.001)$.

Conclusions: The advantages and disadvantages are different for these three surgical techniques. A reasonable operation technique should be adopted considering the patient's unique condition to ensure the stability of hemodynamics.
\end{abstract}

Keywords: Surgical techniques; liver transplantation (LT); classic piggyback; early allograft dysfunction; outflow venous obstruction

Submitted Apr 18, 2021. Accepted for publication Jul 15, 2021. doi: $10.21037 / \mathrm{atm}-21-1945$

View this article at: https://dx.doi.org/10.21037/atm-21-1945

\section{Introduction}

Liver transplantation (LT) is considered the only curative treatment for end stage liver diseases (ESLD) (1). The surgical techniques of LT have continually evolved and have been modified (2). In 1963, Starzl et al. initiated a technique for LT in which the superior (SHVC) and inferior hepatic vena cava (IHVC) and portal vein (PV) were completely blocked, and the retrohepatic inferior vena cava (RHIVC) was removed as a part of the diseased liver; it was named classic OLT (3). However, the hemodynamics in the anhepatic phase is not stable, and reflux of the renal vein is blocked, causing renal dysfunction (4,5). In 1989, Tzakis et al. described a novel technique called classic piggy-back liver transplantation (CPBLT) (6). In this technique, the PHVC is preserved, the SHVC of the donor liver is anastomosed with 
a common opening formed by the left and middle hepatic veins of the recipient liver, and the IHVC of the donor liver is ligated. The flow during the anhepatic period and hemodynamic stability are maintained during the operation. The technique can also reduce the occurrence of renal failure after liver transplantation and is more beneficial to patients with cardiac insufficiency or a poor general condition. However, specific complications related to $\mathrm{CPB}$, such as outflow obstruction, can cause liver congestion, swelling and even delayed graft function (DGF) or transplanted liver failure. In 1992, Belghiti et al. (7) developed the modified piggy-back (MPB) technique in which side-to-side cavocaval anastomosis is performed at the anterior face of the recipient RHIVC to minimize outflow obstruction. Wu et al. (8) also reported another MPB technique, which has the advantages of simplifying the steps of hepatectomy and separation, and the anastomosis of the vena cava is large, thereby avoiding outflow tract obstruction. However, this operation requires a complete blockade of the inferior vena cava, and the anhepatic period is long, thereby leading to intraoperative hemodynamic instability and renal dysfunction.

The CPB and MPB techniques are limited when the caudate lobe of the recipient liver is hypertrophied $(9,10)$. Particularly, in some patients with Budd-Chiari syndrome, the RHIVC is surrounded in the caudate lobe of the liver, making it difficult to preserve the inferior vena cava during hepatectomy $(11,12)$. Additionally, in patients with large liver cancers, the RHIVC tends to be dissected, and classic LT is recommended $(13,14)$. To simplify the operation process and shorten the anhepatic period, we developed a modified classic LT technique (15). In this technique, it is not necessary to isolate the IVC neither dissect the posterior space of the IVC and right adrenal vein. The IVC would be clamped directly from front to back and sutured continually from front to back after dissection of the liver. Compared with conventional classic LT, it is easy to expose and convenient for the surgeon to anastomose.

In general, the selection of surgical techniques depends on the patient's conditions. In this study, we retrospectively analyzed a single-center case series (674 cases) at our center and compared the advantages and disadvantages of each method. We present the following article in accordance with the STROBE checklist (available at https://dx.doi. org/10.21037/atm-21-1945).

\section{Methods}

All procedures were performed in accordance with the ethical standards of the IEC for Clinical Research and Animal Trials of the First Affiliated Hospital of Sun Yat-sen University for human experimentation (institutional and national) and with the Declaration of Helsinki (as revised in 2013). All the organs used in our study were acquired by organ donation, and none were from executed prisoners. The study was approved by the Institutional Ethics Committee for Clinical Research and Animal Trials of the First Affiliated Hospital of Sun Yat-sen University \{Ethical approval ID: [2021]434\}, and an informed consent waiver was granted by the IEC given the retrospective, minimalrisk nature of the study.

In total, 803 patients had undergone transplantation at our department from January 2015 to December 2019. After excluding 112 other types of transplantations (combined liver and kidney transplantation, combined pancreas kidney transplantation, combined liver pancreas transplantation and combined upper abdominal organ transplantation), 686 patients underwent LT were taken into consideration. During the follow-up period, 12 patients were lost to follow up (Figure 1A). Therefore, 674 patients were enrolled in this retrospective study (Figure 1B). The recipient pretransplantation data, including demographics, the results of preoperative laboratory examinations, model for end-stage live disease (MELD) score, Child-Pugh score, history, and diagnosis were collected. The perioperative data, including the anhepatic time, cold ischemia time, duration of operation, blood loss and transfusion of red blood cells (RBCs), fresh frozen plasma (FFP), and platelets, were recorded. Additionally, postoperative surgical complications, length of stay in the intensive care unit (ICU), and the mortality rates and cause of death within the postoperative 30 days were assessed and analyzed.

\section{Surgical techniques}

In general, venous-venous bypass (VVBP) techniques were not used during operation in all groups. The selection of surgical techniques depends on the patient's conditions, including diagnosis, MELD score, pretransplant imaging and laboratory test results.

\section{Modified classic (MC) technique}

The classical surgical technique has been used in our center since 2009. And after that, we developed a modified classic technique which is an improvement of classical orthotopic LT. First, the common bile duct, hepatic artery and portal vein were dissociated in turn for dissection of the first hepatic hilum. 


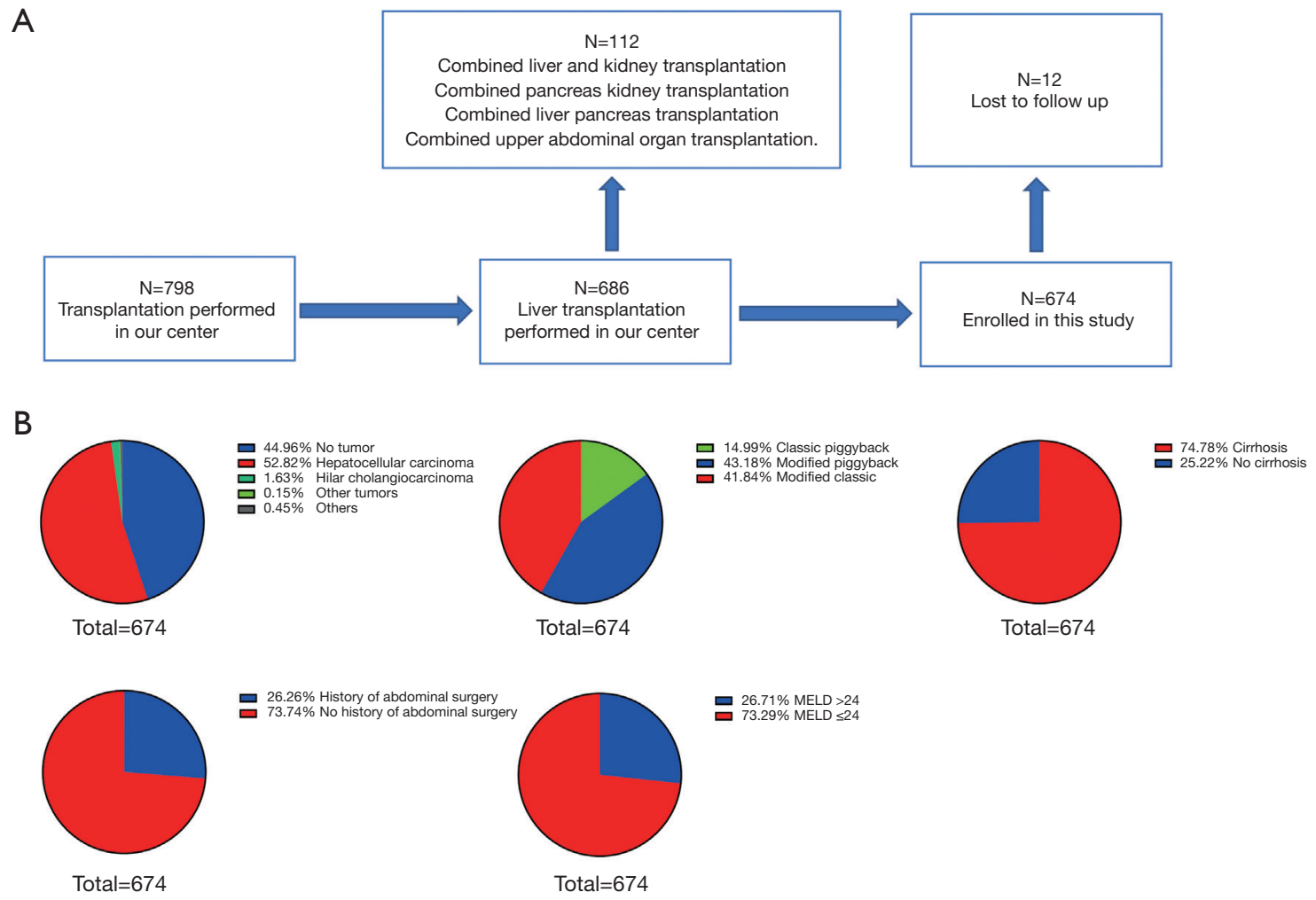

Figure 1 Information about recipient in our study. (A) The study flowchart. (B) Components of participants in our study.

Thereafter, the left triangular ligament, hepatogastric ligament and right triangular ligament were separated and the RHIVC was dissociated. The differences from the conventional method are that dissociating the IVC is unnecessary, and the blocking direction was from front to back. The donor's and recipient's IVC were fixed together at 0 and 6 o'clock using two 4-0 prolene lines. The suture was continuous from back to front. The patients who had undergone this technique were enrolled in MC group (Figure 2A).

\section{MPB technique}

The dissection of the first hepatic hilum and peri-hepatic ligaments was the same as that in the convention technique. Subsequently, the SIVC and PIVC were dissociated and then immediately blocked. The diseased liver was resected close to the anterior wall of the IVC, and all short hepatic veins were ligated. Thereafter, the openings of the three hepatic veins were cut and reshaped, and the anterior wall of the IVC was cut longitudinally to form a large triangular outlet. Anastomosis was performed between the outlet of the recipient IVC and matched the opening of the SVC in the donor liver. The IHVC of the donor liver was ligated before reperfusion. VVBP techniques were not used during operation. The patients who had undergone this technique were enrolled in MPB group (Figure 2B).

\section{CPB technique}

This technique was described as the standard back-table procedure for the liver. The dissection of the first hepatic hilum and peri-hepatic ligaments was the same as that in the conventional technique. The short hepatic veins in the third hepatic hilum were ligated so the right, left and middle hepatic veins (RHV, LHV and MHV) were exposed. The diseased liver was then resected after ligation of the RHV and blockade of the LHV as well as the MHV. Anastomosis was performed between the recipient SVC and the reshaped common trunk of the LHV and MHV of the donor liver. During the entire operation, the blood flow in the IVC was maintained. The patients who had undergone this technique were enrolled in CPB group (Figure 2C).

\section{Postoperative management}

Routine Doppler ultrasound of the liver graft blood flow 

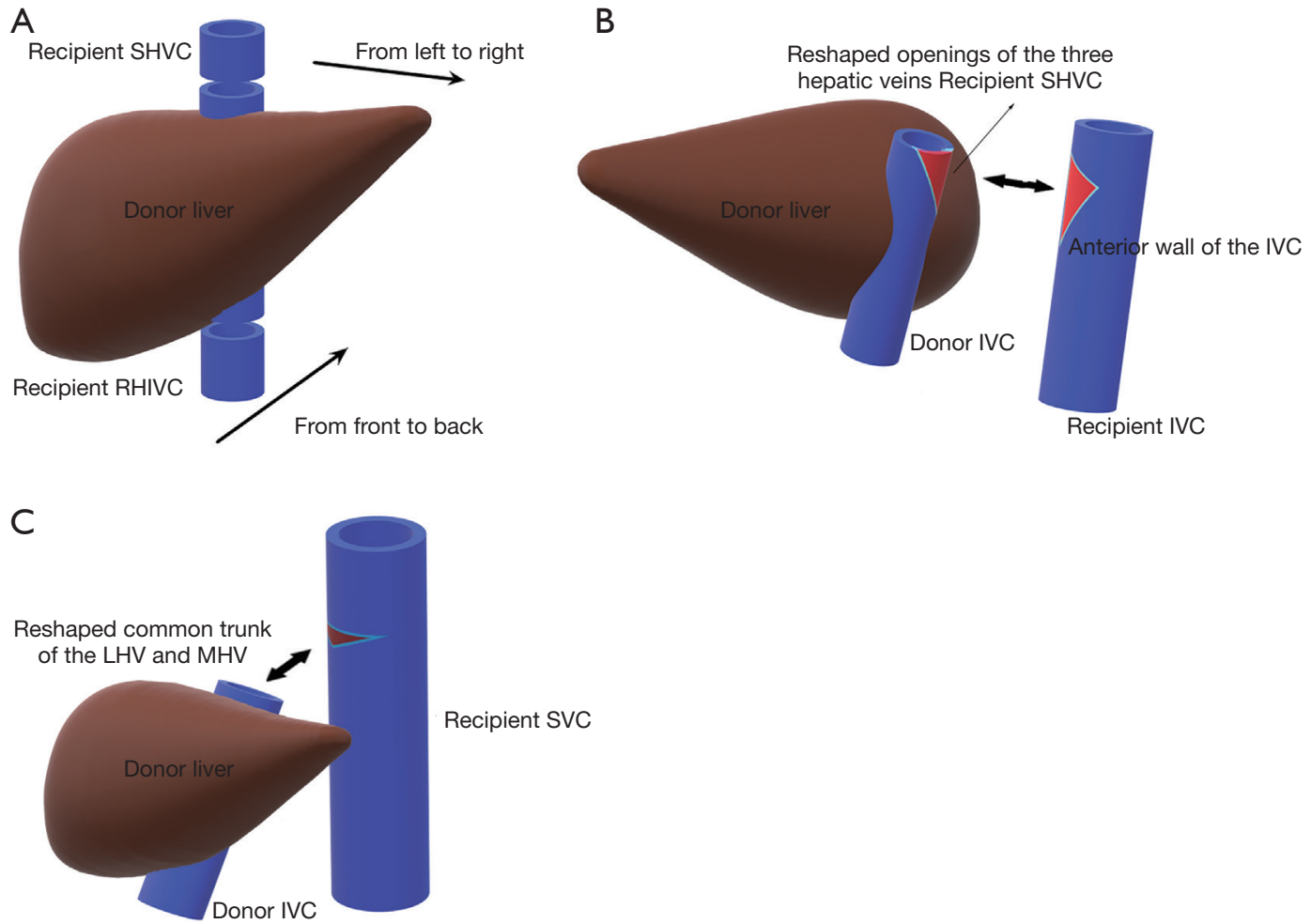

Figure 2 Various surgical techniques in orthotopic liver transplantation. (A) Modified classic; (B) modified piggyback; (C) classic piggyback.

and biliary tract was performed once every 2 days for 7 days. Thereafter, imaging studies were performed based on the patients' clinical status or laboratory findings.

\section{Statistical analysis}

All the statistical analyses of the data were performed using SPSS version 26.0. All the data were expressed as means \pm standard deviation or numbers and percentages of patients. For comparison between groups, chi-square and the ANOVA test were performed for frequencies and continuous data, respectively. Overall survival was compared using the Kaplan-Meier method with a log-rank test. The Cox proportional hazards model was used for multivariate analysis. A P value $<0.05$ was significant.

\section{Results}

\section{Baseline data comparison}

Preoperative data of 674 patients were presented in Figure $1 B$ and Table 1. In general, $54.6 \%$ patients were diagnosed with tumors and $74.8 \%$ patients were diagnosed with cirrhosis. Two-hundred and eighty-two, 291 and 101 patients underwent LT in MC group, MPB group and CPB group, respectively. In comparison, MELD score and Child-Pugh score were significantly higher in $\mathrm{CPB}$ group $(\mathrm{P}=0.008$ and 0.003 , respectively). Except these, the results of other preoperative baseline data including demographic indicators, laboratory test results and past medical history were similar.

\section{Perioperative data comparison}

Intraoperative and postoperative data comparison among different groups were presented in Table 2 and Figure 3. We found that the anhepatic time in MPB group was $57.43 \pm 1.20$ minutes, longer than those in CPB group with $52.88 \pm 1.76$ minutes $(\mathrm{P}<0.05)$.

The operation duration in MPB group were significantly longer than those in $\mathrm{MC}$ group and $\mathrm{CPB}$ group (483.08 \pm 6.98 vs. $455.04 \pm 6.64$ and $448.46 \pm 9.28$ minutes; $\mathrm{P}=0.003$; Figure $3 A$ ). During operation, the volume of blood loss in CPB group was significantly larger than those in $\mathrm{MC}$ group $(2,753.66 \pm 289.65$ vs. $2,116.70 \pm 127.12 \mathrm{~mL} ; \mathrm{P}<0.05$, Figure $3 B)$.

The median ventilation time, duration of oral in-take, 
Table 1 Preoperative data for patients transplanted in different techniques $(\mathrm{n}=674)$

\begin{tabular}{|c|c|c|c|c|c|}
\hline Variable & Total $(n=674)$ & \multicolumn{3}{|c|}{ Surgical techniques } & $\mathrm{P}$ \\
\hline Gender (male/female) & $585 / 89$ & $252 / 30$ & $253 / 38$ & $80 / 21$ & 0.035 \\
\hline Age (ys) & $48.82 \pm 0.50$ & $48.65 \pm 0.77$ & $48.22 \pm 0.80$ & $51.02 \pm 1.11$ & 0.170 \\
\hline Height $(\mathrm{cm})$ & $166.23 \pm 0.54$ & $1.66 .76 \pm 0.84$ & $165.31 \pm 0.93$ & $167.43 \pm 0.72$ & 0.307 \\
\hline Creatinine $(\mu \mathrm{mol} / \mathrm{L})$ & $91.99 \pm 3.31$ & $90.63 \pm 4.15$ & $89.15 \pm 5.04$ & $104.00 \pm 11.89$ & 0.307 \\
\hline Albumin (g/L) & $36.56 \pm 0.55$ & $35.61 \pm 0.43$ & $37.50 \pm 1.17$ & $36.52 \pm 0.81$ & 0.287 \\
\hline Total bilirubin ( $\mu \mathrm{mol} / \mathrm{L})$ & $160.40 \pm 8.20$ & $141.60 \pm 11.50$ & $165.19 \pm 13.14$ & $199.12 \pm 22.68$ & 0.058 \\
\hline MELD score & $17.55 \pm 0.37$ & $16.58 \pm 0.55$ & $17.63 \pm 0.58$ & $20.06 \pm 0.98$ & 0.008 \\
\hline Hypertension, n (\%) & $77(11.4)$ & $38(5.6)$ & $31(4.6)$ & $8(1.2)$ & 0.277 \\
\hline Diabetes, n (\%) & $86(12.8)$ & $42(6.2)$ & $34(5.0)$ & $10(1.5)$ & 0.333 \\
\hline Previous abdominal surgery, $\mathrm{n}(\%)$ & $177(26.3)$ & $74(11.0)$ & $86(12.8)$ & $17(2.5)$ & 0.044 \\
\hline
\end{tabular}

MC, modified classic; MPB, modified piggyback; CPB, classic piggyback; MELD, Model for End-Stage Liver Disease.

ICU stay time and hospital stay time of all patients was 14 hours, 34.5 hours, 100 hours and 24 days, respectively. During comparison, the ventilation time and ICU stay time in $\mathrm{CPB}$ group was both significantly longer than those in $\mathrm{MC}$ group $(\mathrm{P}<0.05$; Figure $3 C)$. The most frequent postoperative complications within 30 days in all patients was abdominal bleeding $(\mathrm{n}=35,5.2 \%)$, followed by HAT $(\mathrm{n}=30,4.5 \%)$ and portal vein thrombosis (PVT; $\mathrm{n}=7,1.0 \%)$. Notably, 3 patients had outflow obstruction (MPB group: $\mathrm{n}=2,0.33 \%$; $\mathrm{CPB}$ group: $\mathrm{n}=1,0.84 \% ; \mathrm{P}=0.035$ ). No significantly differences were observed in other complications (Figure 3D). Ten patients developed primary non-function (PNF) and 131 patients developed early allograft dysfunction (EAD). The 30- and 90-day mortality rate was $8.0 \%$ and $11.3 \%$, respectively. Comparisons among groups showed that the results were similar and without significantly different (Figure 3E).

\section{Survival and multivariable analysis}

Kaplan-Meier survival analyses were performed among groups and presented in Figure 4. In total, one-year survival rates in three groups were similar $(79.7 \%, 83.1 \%$ and $81.1 \%$ in MC, MPB and CPB group, respectively). However, the comparison of 3-year survival rate showed that the overall survival in MPB group were better than those in MC group and $\mathrm{CPB}$ group (3-year: $59 \%, 66 \%$ and $60 \%$ in $\mathrm{MC}, \mathrm{CPB}$ and MPB group, respectively; $\mathrm{P}<0.001$, Figure $4 A$ ). KaplanMeier survival analyses for clinical subgroups were also performed and shown in Figure 4B-4G,4I. In patients with preoperative creatine $>120 \mu \mathrm{mol} / \mathrm{L}$, the overall survival in MC group was worst (1-year: $61.7 \%$; 3-year: $28.6 \%$; $\mathrm{P}<0.001$, Figure $4 G$ ). In patients with high MELD score ( $>24)$, the overall survival in MPB group tended to be the best compared with those in MC group and $\mathrm{CPB}$ group (1-year: $74.4 \%$; 3-year: $60.8 \%$; $\mathrm{P}<0.001$, Figure $4 E$ ). In other subgroups, the results showed that the overall survival were similar in MC group and MPB group and worse in CPB group (Figure 4B-4F,4H). In multivariable analysis, we found that tumor and EAD were both independent factors impacting survival within 30 days and within 90 days, respectively (Table 3).

\section{Discussion}

Many factors play an important role in the outcome of LT and technique-related factors, including operative duration, intraoperative bleeding, and complications, are the most important. In China, majority of patients are complicated with portal hypertension, causing extensive 
Table 2 Perioperative characteristics of patients transplanted in different techniques $(\mathrm{n}=674)$

\begin{tabular}{|c|c|c|c|c|c|c|}
\hline Variable & Total $(n=674)$ & Median & \multicolumn{3}{|c|}{ Surgical techniques } & $\mathrm{P}$ \\
\hline Anhepatic time (min) & $56.16 \pm 0.76$ & 54.00 & $56.01 \pm 1.17$ & $57.43 \pm 1.20$ & $52.88 \pm 1.76$ & 0.136 \\
\hline Cold ischemia time (min) & $416.88 \pm 6.96$ & 416.50 & $409.63 \pm 11.43$ & $431.53 \pm 9.35$ & $394.91 \pm 20.19$ & 0.146 \\
\hline Operation duration (min) & $466.16 \pm 4.36$ & 450.00 & $455.04 \pm 6.64$ & $483.08 \pm 6.98$ & $448.46 \pm 9.28$ & 0.003 \\
\hline FFP volume (mL) & $1,978.74 \pm 56.73$ & $1,600.00$ & $2,033.05 \pm 82.07$ & $1,980.00 \pm 95.37$ & $1,823.47 \pm 123.96$ & 0.472 \\
\hline Platelet volume (u) & $7.57 \pm 2.16$ & 0 & $9.93 \pm 4.41$ & $5.88 \pm 2.37$ & $5.88 \pm 3.19$ & 0.653 \\
\hline Blood loss (mL) & $2,368.07 \pm 95.54$ & $1,500.00$ & $2,116.70 \pm 127.12$ & $2,477.84 \pm 153.03$ & $2,753.66 \pm 289.65$ & 0.052 \\
\hline Ventilation time (hr) & $44.99 \pm 8.00$ & 14.00 & $36.22 \pm 4.18$ & $40.11 \pm 4.32$ & $84.36 \pm 51.46$ & 0.120 \\
\hline Abdominal bleeding, $\mathrm{n}(\%)$ & $35(5.2)$ & - & $17(2.5)$ & $12(1.8)$ & $6(0.9)$ & 0.552 \\
\hline HAT, n (\%) & $30(4.5)$ & - & $14(2.1)$ & $14(2.1)$ & $2(0.3)$ & 0.425 \\
\hline PVT, n (\%) & $7(1.0)$ & - & $1(0.1)$ & $4(0.6)$ & $2(0.3)$ & 0.290 \\
\hline Outflow obstruction, n (\%) & $3(0.4)$ & - & 0 & $1(0.1)$ & $2(0.3)$ & 0.035 \\
\hline AKI, n (\%) & $27(4.0)$ & - & $11(1.6)$ & $11(1.6)$ & $5(0.7)$ & 0.869 \\
\hline PNF, n (\%) & $10(1.5)$ & - & $5(0.7)$ & $4(0.6)$ & $1(0.1)$ & 0.838 \\
\hline EAD, n (\%) & $131(19.4)$ & - & $58(8.6)$ & $49(7.3)$ & $24(3.6)$ & 0.260 \\
\hline Hospital stays $(d)$ & $29.07 \pm 21.01$ & 24.00 & $30.52 \pm 1.65$ & $28.82 \pm 1.01$ & $25.71 \pm 1.38$ & 0.166 \\
\hline
\end{tabular}

MC, modified classic; MPB, modified piggyback; CPB, classic piggyback; RBC, red blood cell; FFP, Fresh Frozen Plasma. ICU, intensive care unit; HAT, hepatic artery thrombosis; PVT, portal vein thrombosis; PNF, primary nonfunction; EAD, early allograft dysfunction; AKI, acute kidney injury.

collateral circulation between the PV and vena cava $(16,17)$. Therefore, hemodynamic instability, such as bleeding during the operation, higher PV pressure during the anhepatic period and lower peripheral vascular resistance, more commonly occur and affect the success of the operation and posttransplant survival. Over the decades, the surgical techniques of LT have continually evolved and have been modified. In summary, the difference among the techniques mainly concerns whether to block the RIHVC, which is the main factor affecting the hemodynamics in the anhepatic phase. In this study, we retrospectively analyzed the data of patients who had undergone LT at our center and compared the advantages and disadvantages of each method.
Many patients are diagnosed with liver cirrhosis in China, and the annual incidence is $2-10 \%(18,19)$. The annual incidence of HCC in patients with liver cirrhosis is $3 \%$ to $6 \%(20)$. In our study, $54.6 \%$ patients were diagnosed with tumors and $74.8 \%$ patients were diagnosed with cirrhosis. MPB would be the optimal choice for patients with nontumor-related ESLD. A large proportion of the tumors in the advanced-stage patients tend to undergo surgical resection or conservative treatment due to economic or ideological reasons, even if the tumors are detected early. LT would be considered only when other treatments were ineffective or if the tumor progressed (21). Thus, the modified classic OLT would be more radical.

The median operation duration at our center was 
A
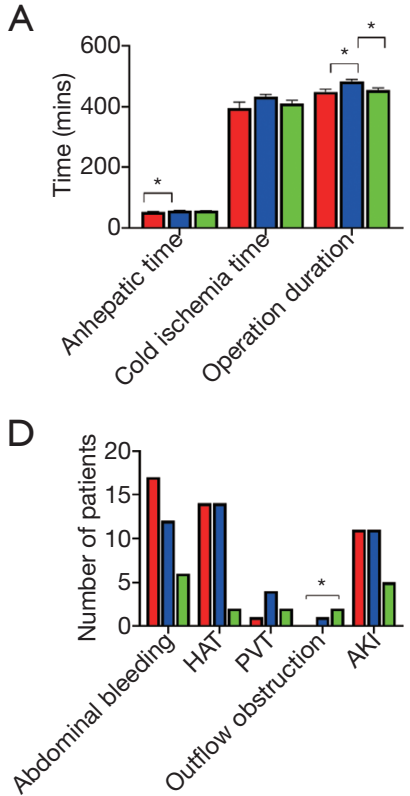
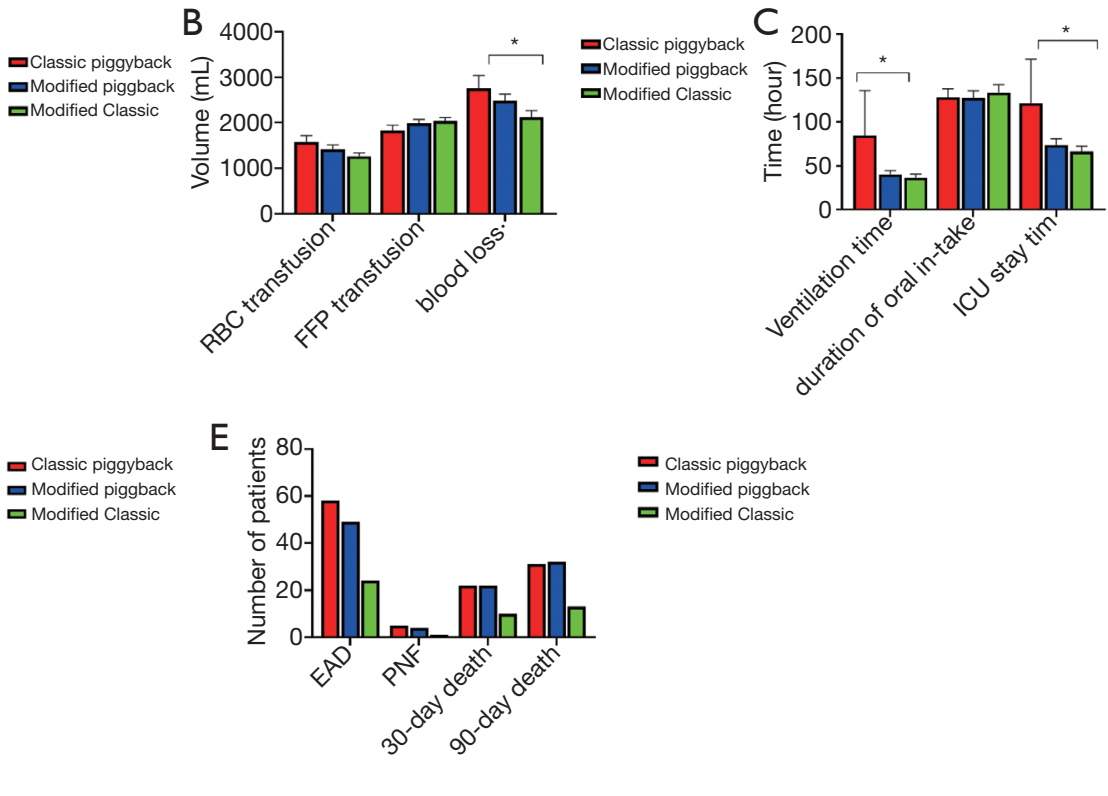

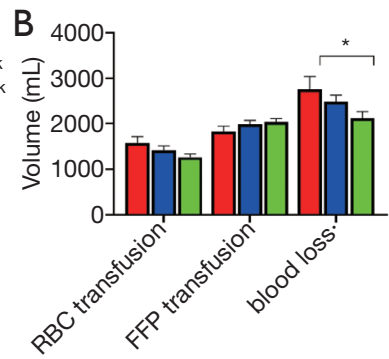

$\mathrm{E}$
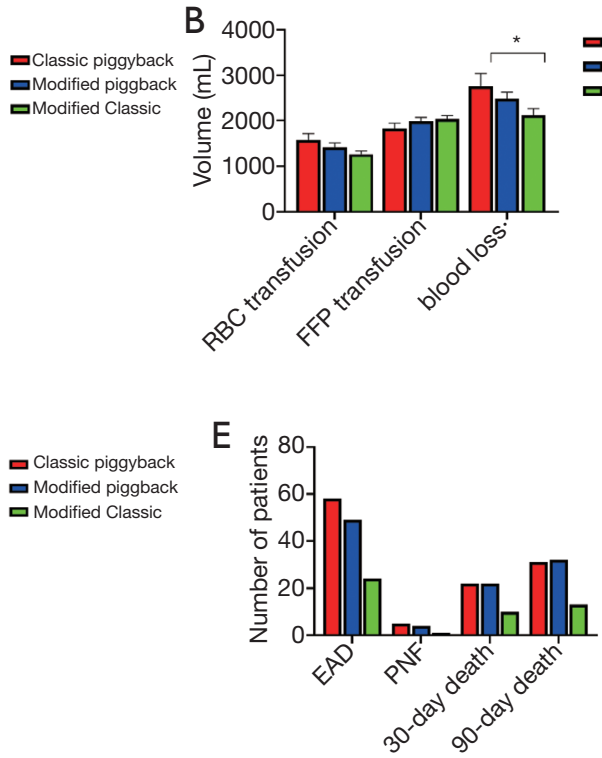

口Classic piggyback - Modified piggback 口Modified Classic

Figure 3 Perioperative comparison in three groups. (A) Anhepatic time, cold ischemia time and operation time (B) RBC transfusion, FFP transfusion and blood loss; (C) ventilation time, duration of oral in-take and ICU stay time; (D) abdominal bleeding, HAT, PVT, outflow obstruction and AKI; (E) EAD, PNF, 30- and 90-day death. *means $\mathrm{P}<0.05$. FFP, fresh frozen plasma; ICU, intensive care unit; HAT, hepatic artery thrombosis; PVT, portal vein thrombosis; AKI, acute kidney injury; EAD, early allograft dysfunction; PNF, primary nonfunction.

450 minutes. Compared with previous national reports (range from 320 to 708 minutes) (22-25), our data for the MPB technique $(483.08 \pm 6.98$ minutes) in MPB group were consistent with the international data and were significantly longer than those in $\mathrm{MC}$ group and $\mathrm{CPB}$ group. This result is acceptable compared with that in the randomized trial conducted by Jovine et al. (26) in which the operative time was $506 \pm 85$ minutes and $462 \pm 87$ minutes in the $\mathrm{CPB}$ and conventional groups, respectively. The cold ischemia time is an inherent and unavoidable factor in LT (27). Ruiz de Azúa-López et al. (28) reported that a long ischemia time ( $>6$ hours) leads to a higher rate of complications after LT. The median cold ischemia time in this study was 416.5 minutes and comparable with that in previous reports (29). The anhepatic phase is defined as the time from the dissection of the recipient liver to reperfusion of the graft. Cleland et al. (30) concluded that the anhepatic phase would increase blood loss because of the absence of hepatic synthesis and clearance. Ijtsma et al. (31) revealed that a long anhepatic phase duration ( $>100$ minutes) is an independent risk factor for graft dysfunction in LT. VVBP (32) was introduced to solve the problems of interruption of venous return, hemodynamic instability, and renal failure (33) caused by a full or partial cross-clamping of the inferior vena cava. In our study, VVBP was not used in all groups. Because of the shortening of anhepatic time therefore the decrease of hemodynamic instability, VVBP is not commonly used in LT with MC; In addition, In MPB and CPB, because inferior vena cava is not blocked, VVBP is not needed, neither. The median and mean anhepatic times were 54 and $56.16 \pm 0.76$ minutes, respectively. This result may suggest that the modified classic LT technique could achieve comparable anhepatic times as CPB or MPB because of its simplified procedure compared with the classic technique.

According to our study, the median intraoperative blood loss, transfusion of RBCs, and FFP were 1,500, 1,000 and $1,600 \mathrm{~mL}$, respectively. A significant large volume of blood loss was founded in CPB group and was within an acceptable range compared with the experience at other centers $(34,35)$. In a cohort study of over 5,000 patients in France, Savier et al. (36) found that the median ICU duration after LT was 8 days (5-15 days). Mehrabi et al. (24) reported a 14-day ICU and IMC stay in their experience of 500 LTs using the MPB technique. In our study, the median 

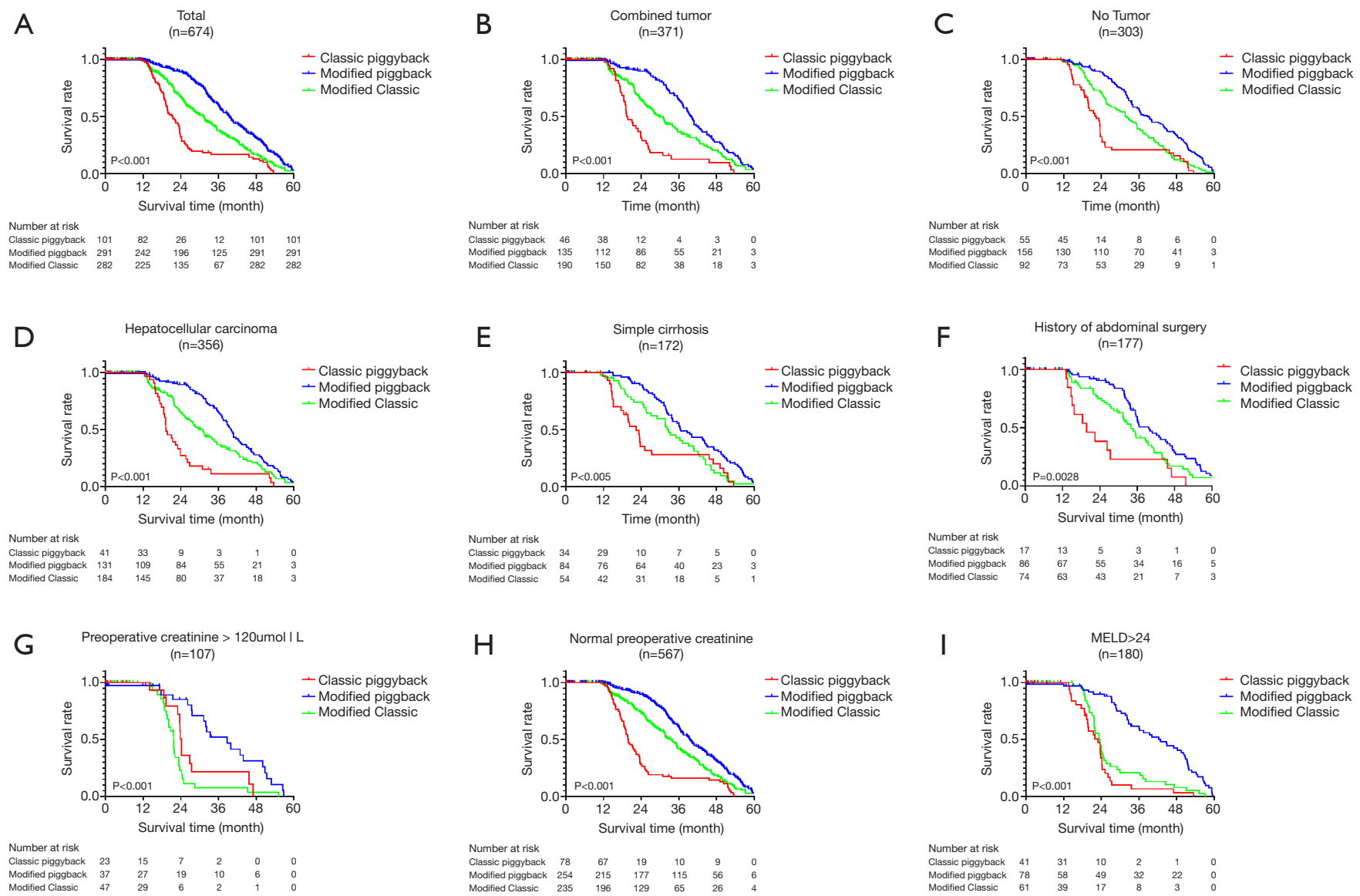

Figure 4 Kaplan-Meier survival analyses in total and subgroups. (A) Total; (B) combined tumor; (C) no tumor; (D) hepatocellular carcinoma; (E) simple cirrhosis; (F) history of abdominal surgery; (G) preoperative creatinine $>120 \mu \mathrm{mol} / \mathrm{L}$; (H) normal preoperative creatinine; (I) MELD > 24. MELD, model for end stage liver disease.

and mean ICU stay time were 100 and $77.63 \pm 8.67$ hours, respectively, markedly shorter than previous experiences. Our center promoted the concept of 'enhanced recovery after surgery' (ERAS) to achieve early extubation, early functional exercise, and a shortened length of stay at the hospital (37).

Complications in the early postoperative period are important concerns in LT. AKI was a prevalent complication, and its incidence was $4.0 \%$ in our study $(38,39)$. The incidence of AKI in previous reports ranged from $0 \%$ to $16.7 \%(40,41)$. AKI was mainly caused by hemodynamic instability, IVC blockade, and severe intraoperative blood loss. Hesse et al. (40) showed that the incidence of postoperative renal dysfunction was significantly lower in the MPB group. However, no significant difference was found in the incidence of renal failure among the different groups in our study. Although the IVC was blocked during the operation in MC group, the operation process was simplified and the operative time was shortened, leading to little impact on the hemodynamic stability (42). However, in patients with high preoperative creatinine, the overall survival in MC group was worst. The results suggest that piggyback is a better choice for patients with poor preoperative renal function. Notably, outflow obstruction occurred in both MPB and CPB group, with incidences of $0.3 \%$ and $2.0 \%$, respectively. Arudchelvam et al. (43) reported that $2.0 \%$ of 253 patients with CPB had outflow obstruction. An enlarged caudate lobe, oversized donor graft, abnormalities of the IVC, or adhesions between the liver and the RHIVC may be the main obstacles for the use of the CPB or MPB technique (44). In our center, the donor IVC was trimmed in a triangle-shaped fashion to avoid obstruction of hepatic venous outflow. Additionally, the incision on the IVC should have sufficient distance 
Table 3 Multivariant analysis on cause of death within 30 days and 90 days patients transplanted with different techniques

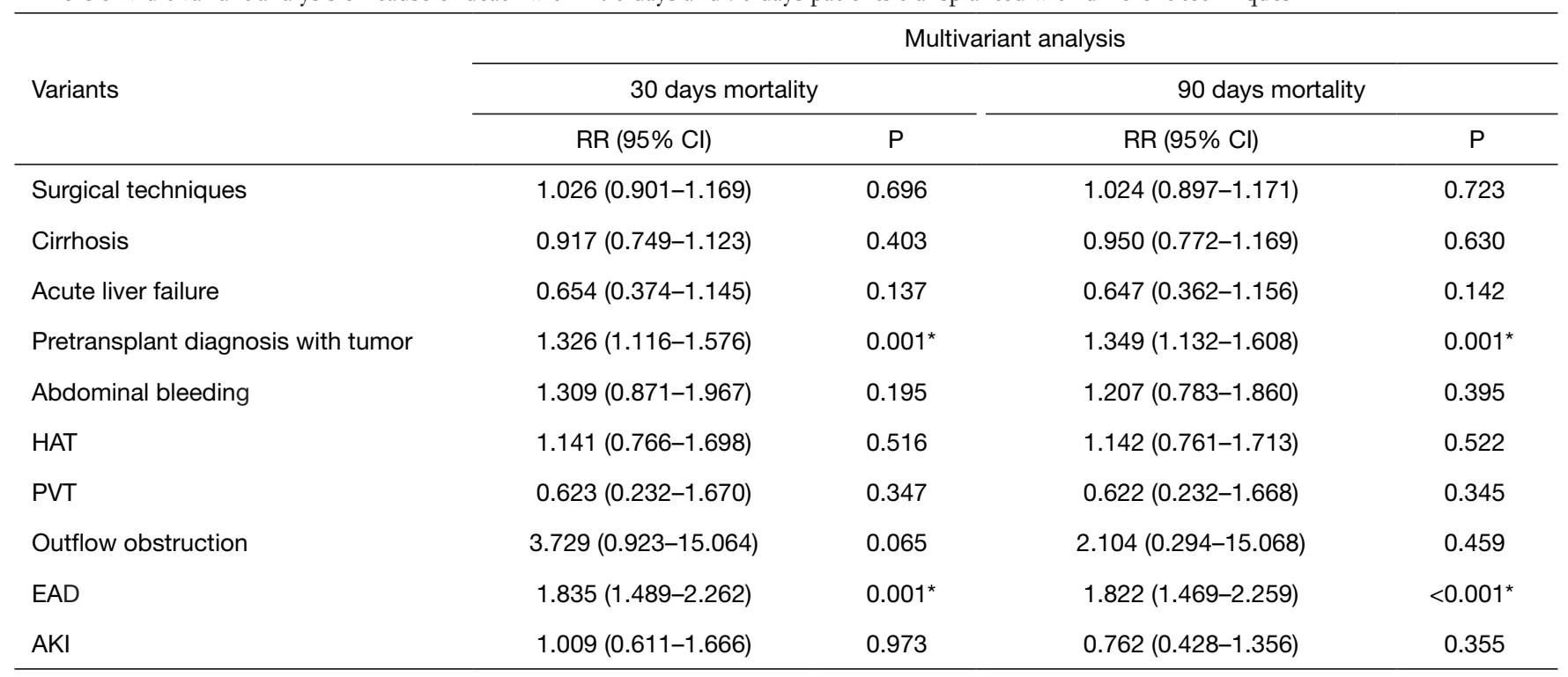

${ }^{*} \mathrm{P}<0.05$. MC, modified classic; MPB, modified piggyback; CPB, classic piggyback; HAT, hepatic artery thrombosis; PVT, portal vein thrombosis; PNF, primary nonfunction; EAD, early allograft dysfunction; AKI, acute kidney injury.

from the hepatic veins. The early (within 30 days) and intermediate (within 90 days) mortality rates in all cases were $8.0 \%$ and $11.3 \%$, respectively. In a previous report, approximately $10.9 \%$ had graft failure within 30 days (45). In another study, Zanetto et al. reported the estimated 30-day mortality rate of over 3,000 LTs was $8.4 \%$ (46). For longterm survival analysis, the overall survival in MC group and MPB group were similar and better than those in CPB group in total. From results of subgroups we figured out that in patients with severe ESLD (MELD >24), MPB would be the optimal choice.

Our study has limitations. The study is a retrospective study from a single center. Selection bias exists and may impact the comparison results. A summary of Multi center experience or a randomized clinical trial is needed.

\section{Conclusions}

Our study demonstrates that different surgical techniques have specific advantages and disadvantages and a reasonable operation technique based on the patient's condition to ensure the stability of hemodynamics during the operation is of considerable significance to improve the prognosis. In brief, in patients with poor pretransplant renal function, the MC technic would not be recommended. In patients with high MELD score (>24), MPB tended to be the best choice. In other conditions, MPB or MC would have similar outcomes.

\section{Acknowledgments}

Funding: This work was supported by the National Natural Science Foundation of China (81401324 and 81770410), Guangdong Basic and Applied Basic Research Foundation (2020A1515011557), China and the funders (Xiaoshun $\mathrm{He}$ and Maogen Chen) were both corresponded for this study.

\section{Footnote}

Reporting Checklist: The authors have completed the STROBE reporting checklist. Available at https://dx.doi. org/10.21037/atm-21-1945

Data Sharing Statement: Available at https://dx.doi. org/10.21037/atm-21-1945

Peer Review File: Available at https://dx.doi.org/10.21037/ atm-21-1945

Conflicts of Interest: All authors have completed the ICMJE uniform disclosure form (available at https://dx.doi. org/10.21037/atm-21-1945). The authors have no conflicts of interest to declare.

Ethical Statement: The authors are accountable for all aspects of the work in ensuring that questions related 
to the accuracy or integrity of any part of the work are appropriately investigated and resolved. All procedures were performed in accordance with the ethical standards of the IEC for Clinical Research and Animal Trials of the First Affiliated Hospital of Sun Yat-sen University for human experimentation (institutional and national) and with the Declaration of Helsinki (as revised in 2013). All the organs used in our study were acquired by organ donation, and none were from executed prisoners. The study was approved by the Institutional Ethics Committee for Clinical Research and Animal Trials of the First Affiliated Hospital of Sun Yat-sen University (Ethical approval ID: [2021]434), and an informed consent waiver was granted by the IEC given the retrospective, minimal-risk nature of the study.

Open Access Statement: This is an Open Access article distributed in accordance with the Creative Commons Attribution-NonCommercial-NoDerivs 4.0 International License (CC BY-NC-ND 4.0), which permits the noncommercial replication and distribution of the article with the strict proviso that no changes or edits are made and the original work is properly cited (including links to both the formal publication through the relevant DOI and the license). See: https://creativecommons.org/licenses/by-nc-nd/4.0/.

\section{References}

1. Morris PJ. Transplantation--a medical miracle of the 20th century. N Engl J Med 2004;351:2678-80.

2. Müller PC, Kabacam G, Vibert E, et al. Current status of liver transplantation in Europe. Int J Surg 2020;82S:22-9.

3. Starzl TE, Marchioro TL, Porter KA, et al. Homotransplantation of the liver. Transplantation 1967;5:Suppl:790-803.

4. Estrin JA, Belani KG, Ascher NL, et al. Hemodynamic changes on clamping and unclamping of major vessels during liver transplantation. Transplant Proc 1989;21:3500-5.

5. Lapisatepun W, Lapisatepun W, Agopian V, et al. Venovenous Bypass During Liver Transplantation: A New Look at an Old Technique. Transplant Proc 2020;52:905-9.

6. Tzakis A, Todo S, Starzl TE. Orthotopic liver transplantation with preservation of the inferior vena cava. Ann Surg 1989;210:649-52.

7. Belghiti J, Panis Y, Sauvanet A, et al. A new technique of side to side caval anastomosis during orthotopic hepatic transplantation without inferior vena caval occlusion. Surg
Gynecol Obstet 1992;175:270-2.

8. Wu YM, Voigt M, Rayhill S, et al. Suprahepatic venacavaplasty (cavaplasty) with retrohepatic cava extension in liver transplantation: experience with first 115 cases. Transplantation 2001;72:1389-94.

9. Busque S, Esquivel CO, Concepcion W, et al. Experience with the piggyback technique without caval occlusion in adult orthotopic liver transplantation. Transplantation 1998;65:77-82.

10. Pratschke S, Rauch A, Albertsmeier M, et al. Temporary Intraoperative Porto-Caval Shunts in Piggy-Back Liver Transplantation Reduce Intraoperative Blood Loss and Improve Postoperative Transaminases and Renal Function: A Meta-Analysis. World J Surg 2016;40:2988-98.

11. Navarro F, Le Moine MC, Fabre JM, et al. Specific vascular complications of orthotopic liver transplantation with preservation of the retrohepatic vena cava: review of 1361 cases. Transplantation 1999;68:646-50.

12. Akamatsu N, Sugawara Y, Kokudo N. Budd-Chiari syndrome and liver transplantation. Intractable Rare Dis Res 2015;4:24-32.

13. Lerut J, Ciccarelli O, Roggen F, et al. Cavocaval adult liver transplantation and retransplantation without venovenous bypass and without portocaval shunting: a prospective feasibility study in adult liver transplantation. Transplantation 2003;75:1740-5.

14. Gunasekaran G, Bekki Y, Lourdusamy V, et al. Surgical Treatments of Hepatobiliary Cancers. Hepatology 2021;73 Suppl 1:128-36.

15. He XS, Wu LW, Ju WQ, et al. Selection of four surgical techniques in orthotopic liver transplantation. Zhongguo Yi Xue Ke Xue Yuan Xue Bao 2008;30:426-9.

16. Kong L, Lv T, Jiang L, et al. Outcomes of hemi- versus whole liver transplantation in patients from mainland china with high model for end-stage liver disease scores: a matched analysis. BMC Surg 2020;20:290.

17. Xiao J, Wang F, Wong NK, et al. Global liver disease burdens and research trends: Analysis from a Chinese perspective. J Hepatol 2019;71:212-21.

18. Chinese Society of Infectious Diseases, Chinese Medical Association; Chinese Society of Hepatology, Chinese Medical Association. The guidelines of prevention and treatment for chronic hepatitis B (2019 version). Zhonghua Gan Zang Bing Za Zhi 2019;27:938-61.

19. Peng Y, Qi X, Guo X. Child-Pugh Versus MELD Score for the Assessment of Prognosis in Liver Cirrhosis: A Systematic Review and Meta-Analysis of Observational 
Studies. Medicine (Baltimore) 2016;95:e2877.

20. Fattovich G, Bortolotti F, Donato F. Natural history of chronic hepatitis $\mathrm{B}$ : special emphasis on disease progression and prognostic factors. J Hepatol 2008;48:335-52.

21. Chen Z, Lin X, Chen C, et al. Analysis of preoperative circulating tumor cells for recurrence in patients with hepatocellular carcinoma after liver transplantation. Ann Transl Med 2020;8:1067.

22. Zieniewicz K, Krawczyk M, Nyckowski P, et al. Liver transplantation: comparison of the classical orthotopic and piggyback techniques. Transplant Proc 2002;34:625-7.

23. Durand F, Aschehoug J, Sauvanet A, et al. Preservation of renal perfusion and postoperative renal function by side-to-side cavo-caval anastomosis in liver transplant recipients. Transpl Int 1995;8:407-10.

24. Mehrabi A, Mood ZA, Fonouni H, et al. A singlecenter experience of 500 liver transplants using the modified piggyback technique by Belghiti. Liver Transpl 2009;15:466-74.

25. Kim HY, Ko JS, Joh J, et al. Weaning of Veno-venous Bypass in Liver Transplantation: A Single Center Experience. Transplant Proc 2018;50:2657-60.

26. Jovine E, Mazziotti A, Grazi GL, et al. Piggy-back versus conventional technique in liver transplantation: report of a randomized trial. Transpl Int 1997;10:109-12.

27. Paterno F, Guarrera JV, Wima K, et al. Clinical Implications of Donor Warm and Cold Ischemia Time in Donor After Circulatory Death Liver Transplantation. Liver Transpl 2019;25:1342-52.

28. Ruiz de Azúa-López Z, Naranjo-Izurieta JR, Lameirao J, et al. Cold Ischemia Time as a Factor in Post-transplantation Complications for Orthotopic Hepatic Transplantation. Transplant Proc 2018;50:637-9.

29. Chen XB, Xu MQ. Primary graft dysfunction after liver transplantation. Hepatobiliary Pancreat Dis Int 2014;13:125-37.

30. Cleland S, Corredor C, Ye JJ, et al. Massive haemorrhage in liver transplantation: Consequences, prediction and management. World J Transplant 2016;6:291-305.

31. Ijtsma AJ, van der Hilst CS, de Boer MT, et al. The clinical relevance of the anhepatic phase during liver transplantation. Liver Transpl 2009;15:1050-5.

32. Shaw BW Jr, Martin DJ, Marquez JM, et al. Venous bypass in clinical liver transplantation. Ann Surg 1984;200:524-34.

33. Fonouni H, Mehrabi A, Soleimani M, et al. The need for venovenous bypass in liver transplantation. HPB (Oxford) 2008;10:196-203.

34. Wang HZ, Dong JH, Wang SG, et al. Modified hepatic outflow tract reconstruction in piggyback liver transplantation. Hepatobiliary Pancreat Dis Int 2003;2:206-10.

35. Salizzoni M, Andorno E, Bossuto E, et al. Piggyback techniques versus classical technique in orthotopic liver transplantation: a review of 75 cases. Transplant Proc 1994;26:3552-3.

36. Savier E, Brustia R, Golmard JL, et al. Influence of 4 preservation solutions on ICU stay, graft and patient survival following liver transplantation. J Visc Surg 2020;157:87-97.

37. Khalil A, Ganesh S, Hughes C, et al. Evaluation of the enhanced recovery after surgery protocol in living liver donors. Clin Transplant 2018;32:e13342.

38. Aguayo E, Hadaya J, Nakhla M, et al. Outcomes and resource use for liver transplantation in the United States: Insights from the 2009-2017 National Inpatient Sample. Clin Transplant 2021;35:e14262.

39. Tovikkai C, Limsrichamrern S, Dumronggittigule $W$, et al. Delayed Calcineurin Inhibitor Introduction Without Antibody Induction in Liver Transplantation Is Safe and Helps Preserve Kidney Function. Transplant Proc 2021;53:645-8.

40. Hesse UJ, Berrevoet F, Troisi R, et al. Hepato-venous reconstruction in orthotopic liver transplantation with preservation of the recipients' inferior vena cava and veno-venous bypass. Langenbecks Arch Surg 2000;385:350-6.

41. Lee HC, Yoon SB, Yang SM, et al. Prediction of Acute Kidney Injury after Liver Transplantation: Machine Learning Approaches vs. Logistic Regression Model. J Clin Med 2018;7:428.

42. Lebrón Gallardo M, Herrera Gutierrez ME, Seller Pérez G, et al. Risk factors for renal dysfunction in the postoperative course of liver transplant. Liver Transpl 2004;10:1379-85.

43. Arudchelvam J, Bartlett A, McCall J, et al. Hepatic venous outflow obstruction in piggyback liver transplantation: single centre experience. ANZ J Surg 2017;87:182-5.

44. Steib A, Saada A, Clever B, et al. Orthotopic liver transplantation with preservation of portocaval flow compared with venovenous bypass. Liver Transpl Surg 1997;3:518-25.

45. Belghiti J, Ettorre GM, Durand F, et al. Feasibility 
and limits of caval-flow preservation during liver transplantation. Liver Transpl 2001;7:983-7.

46. Zanetto A, Rodriguez-Kastro KI, Germani G, et al.
Mortality in liver transplant recipients with portal vein thrombosis - an updated meta-analysis. Transpl Int 2018;31:1318-29.

Cite this article as: Chen Z, Ju W, Chen C, Wang T, Yu J, Hong X, Dong Y, Chen M, He X. Application of various surgical techniques in liver transplantation: a retrospective study. Ann Transl Med 2021;9(17):1367. doi: 10.21037/atm-21-1945 